Colvin, Alexander (2001): Gerechtigkeit ohne Gewerkschaft und Betriebsrat? Konfliktschlichtung in gewerkschaftsfreien Betrieben in den USA, in: WSI-Mitteilungen, 54.Jg., H.12, S.743- 749.

Gorz, André (2004): Wissen, Wert und Kapital. Zur Kritik der Wissensökonomie, Zürich: Rotpunktverlag.

Jaehrling, Karen, Kalina, Thorsten, Vanselow, Achim, Voss-Dahm, Dorothea (2006): Niedriglohnarbeit in der Praxis - Arbeit in Häppchen für wenig Geld. In: Sterkel, G., Schulten, T. Wiedemuth, J. (Hg.): Mindestlöhne gegen Lohndumping. Hamburg, S.114-141.

Keller, Berndt, Seifert, Hartmut (2006): Atypische Beschäftigungsverhältnisse: Flexibilität, soziale Sicherheit und Prekarität, in: WSI Mitteilungen, 59. Jg., H.5, S.235-240.

Krell, Gertraude (1994): Vergemeinschaftende Personalpolitik, München/Mering: Rainer Hampp. Mabrouki, Abdel (2004): Génération Précaire, Paris: le cherche midi.

Meyer, Malte (2002): Neuanfang in der Defensive. US-Gewerkschaften unter Handlungsdruck, Hamburg: VSA.

Nölting, Nils J. (2004): Betriebsratsarbeit im Niedriglohnsektor. Eine Fallstudie in der Systemgastronomie. Eine Veröffentlichung der Kooperationsstelle Wissenschaft - Arbeitswelt im Landesinstitut Sozialforschungsstelle kowa, Dortmund, Juni 2004, www.kowa-dortmund.de/ docs/veroeff/br-arbeit.pdf.

Perrin, E., Peroumal, F., avec la participation de Contrepois, S. (2007): Luttes et syndicalisme dans la restauration rapide parisienne, in: Bouffartigue, P., Pendaries, J.-R., Peroumal, F., Perrin, $\mathrm{E}$. Action collective et précrités. Le syndicalisme i l'épreuve Rapport final pour hin, E. Action collective et $P$ CNRS- Université de la Méditerranée-Université de Provence, Mars 2007, pp.125-181.

Royle, Tony (2000): Working for McDonald's in Europe. London/New York.

Puech, Isabel (2004): Le temps du remue-ménage. Conditions d'emploi et de travail de emmes de chambre, in: Sociologie du travail 46 (2004), S.150-167.

Schäfer, Claus (2006): Der Niedriglohnsektor in der Verteilungsfalle, in: Sterkel, Gabriele, Schulten, Thorsten, Wiedemuth Jörg (Hg.), Mindestlöhne gegen Lohndumping. Hamburg: VSA, S.35-60.

Schmidt, Rudi (2007): Belegschaft als Objekt. Unternehmerische Integrationsstrategien in interessentheoretischer Perspektive, in: Benthin, R., Brinkmann, U. (Hg.), Unternehmenskultur und Mitbestimmung. Frankfurt a.M.New York: Campus.

Tie-Internationales Bildungswerk e.V., AFP e.V., express-Redaltion (Hg.) (2003): Das Solidaritätskollektiv: Eine Erfahrung der etwas anderen Art. Arbeitskämpfe und Organisationsversuche in gewerkschaftlich nicht organisierten Betrieben und Sektoren, Ränkeschmiede, Texte zur Arbeiterlnnenbewegung, No.14, Oktober 2003, Offenbach

Weber, Helène (2005): Les freins à la mobilisation syndicale des salariés chez McDonald's, Xes Jounées Internationales de sociologie du travail, Rouen 24 et 2 novembre 2005, Relations au travail, relations de travail, Vol 1, S. 355-366.
Ingo Matuschek, Frank Kleemann, G. Günter Voß

\section{Subjektivierte Taylorisierung als Beherrschung der Arbeitsperson}

Die sozialwissenschaftliche Diskussion um Entwicklungen der Arbeitswelt setzt taylorisierte Arbeitsformen und vielfältige Modelle indirekt gesteuerter „post-tayloristischer", „entgrenzter" und „subjektivierter" Arbeit zumeist in Opposition zu einander. ${ }^{1}$ Als Belege werden weiterhin stark taylorisierte Tätigkeiten in der industriellen Produktion bzw. vor allem Dienstleister unterschiedlicher Couleur, die zumeist anspruchsvolle Aufgaben weitgehend 'selbstorganisiert' bewältigen, angeführt. Es scheint, als seien die Arbeitsformen gleichsam voneinander abgeschottet.

Nun ist „Selbstorganisation“ (Pongratz/ Voß 1997) bzw. „Selbsträtigkeit" (Wolf 1999) der Arbeitenden, also die situationsadäquate, bisweilen (im Sinne Joas 1992) „kreative" Auslegung formaler Vorgaben eine notwendige Leistung, um Produktionsprozesse (vgl. Weltz 1991) aufrecht zu erhalten. Sie war auch unter tayloristischen Vorzeichen immer schon vonnöten, wurde aber in der Literatur selten thematisiert (vgl. kritisch dazu Böhle 1998, Schumann 2000). Der Bedarf nach „subjekthaftem“" Arbeitshandeln (Baukrowitz/Boes 1996) steigt angesichts der fortschreitenden Informatisierung der $\mathrm{Arbeit}^{2}$ weiter an: Informatisierung erfordert, wie jeder Technisierungsprozess, ergänzende kompensatorische und strukturierende Leistungen der Subjekte (Schimank 1986). Solche Prozesse einer Subjektivierung von Arbeit (vgl. Kleemann et al. 2002) erhalten auch bei nur gering qualifizierten Tätigkeiten erhöhte Bedeutung. Im Zuge der Vermarktlichung von Arbeit (Moldaschl/Sauer 2000) steht zuneh-

1 Entgrenzung manifestiert sich u a in zeitlich, räumlich, sozial, sachlich und emotional wirksamen Folgen perforierter Begrenzungen und Regularien von Arbeit (vgl. dazu Gottschall/Voß 2003; Kratzer 2003; Minssen 2000, Voß 1998). Damit geht die Subjektivierung von Arbeit einher. Dieser Begriff bezeichnet die verstärkte Einbeziehung personaler Qualitäten, zu denen etwa kommunikative Kompetenzen, die Fähigkeit zur Selbstorganisation etc. (vgl. den Überblick bei Kleemann et al. 2002; Kropf 2005) gehören.

2 Informatisierung und Subiekrivierung schließen einander keineswers aus - gleichwohl werden beide Konzepte im arbeits- und industriesoziologischen Diskurs überwiegend als (falsche) Opposition behandelt, indem Informatisierung einseitig als Formalisierung von Arsche) Opposition behandelt, indem Informatisierung einseitig als Formalisierung von A-
beitsabläufen gesehen wird (vgl. dazu Kleemann/Matuschek 2008). Empirisch sind jedoch beitsabläufen gesehen wird (vgl. dazu Kleemann/Matuschek 2008). Empirisch sind jedoch
komplementäre Prozesse der systemischen Einbindung, Entqualifizierung und Formalisierung der Arbeit (Schmiede 1996) zu beobachten.

PROKLA. Zeitschrift für kritische Sozialwissenschaft, Heft 150, 38. Jg., 2008, Nr. 1, 49-64 
mend jeder einzelne Arbeitsplatz permanent zur Disposition und Marktzwänge werden auch in bislang mittelbar berührten ausführenden Bereichen direkt spürbar: Berufstätige sind vermehrt angehalten, die Erwerbssicherung als ureigenen Teil ihrer Arbeitsaufgabe zu verstehen. In der Praxis geht damit häufig die Erwartung an außergewöhnliche Motivation ("Commitment") einher bis hin zur Aufforderung, bei aller Unterordnung „unternehmerisch“ (Bröckling 2007, ähnlich auch Voß/ Pongratz 1998) zu handeln.

Der Taylorismus (Taylor 1977) als bisher vor allem in ausführenden Bereichen dominante Leitphilosophie für die Arbeitsgestaltung und die Nutzung von Arbeitskraft ist damit aber nicht passé: Aufgaben werden weiterhin mittels kennziffernbasierter Arbeitsorganisation minutiös vorgeformt, Arbeitsabläufe wie -ergebnisse gezielt kontrolliert und individuelle Arbeitsleistungen anhand unterschiedlichster Parameter informationstechnisch überaus detailliert erfasst. Im Ergebnis werden enge betriebliche Kontrolle und erweiterte 'subjektivierte' Selbsttätigkeit $\mathrm{zu}$ einem neuen Organisationsmodus von Arbeit (re-)kombiniert, der auf einen kontrollierten Einsatz der Subjektivität der Arbeitenden abzielt. Diese Gemengelage einer Verbindung tayloristischer Formen rigider Fremdbestimmung mit Elementen selbstorganisierter und dadurch Subjektivität verstärkt nutzender Arbeit wird im Folgenden als subjektivierte Taylorisierung begriffen. Nicht nur die bloße Arbeitskraft, sondern die ganze Arbeitsperson mit ihren subjektiven Fähigkeiten wird arbeitsorganisatorisch interessant. Um trotz größerer Freiräume in der Arbeitsgestaltung die betrieblichen Herrschaftsbeziehungen zu sichern, sind flexibilisierte Formen der Standardisierung notwendig. Das betrifft betriebliche Arbeitszeitregelungen ebenso wie konkrete Arbeitsaufgaben, die Ausrichtung der betrieblichen Führungskultur und die Kontrollformen. Kennzeichen subjektivierter Taylorisierung ist nicht zuletzt eine auf die Einzelnen zielende Ideologie der Vermarktlichung, die nur (variabel definierbaren) Arbeitserfolg, nicht aber die Arbeitsleistung fokussiert. Ein paradigmatisches Exempel zugleich taylorisierter wie subjektivierter Arbeit bieten Call Center. Günter Wallraffs Reportage im ZEIT-Magazin (Wallraff 2007) lenkte den Blick auf die nicht wenigen „Schwarzen Schafe" der Branche, die, darin „Drückerkolonnen“ ähnlich, auf den Verkauf überteuerter Produkte zielen. Gleichwohl stellen seriöse Anbieter, zumeist gekoppelt an große und mittlere Unternehmen bzw. für diese tätig, den Kern der Branche dar. Solche Call-Center mit relativ umfangreichen Beratungsauftrag und In- wie Outbound-Telefonie stehen im Mittelpunkt der folgenden Ausführungen.

Am Beispiel qualifizierter Call-Center-Dienstleistungen von Unternehmen in der Finanzdienstleistungs- und Telekommunikationsbranche sowie Technischen Hotlines gehen wir der Frage nach, inwiefern die hier anzutreffenden Formen der Arbeitsorganisation und des Arbeitshandelns Ausdruck einer subjektivierten Taylorisierung sind und welche Spezifika diese neue Form betrieb- licher Arbeitsorganisation aufweist. ${ }^{3}$ Dazu fokussieren wir zunächst sowohl organisationale Prämissen und betriebliche Bedingungen wie sich eröffnende (relative) Handlungsspielräume für die Subjekte (1). In einem zweiten Schritt erörtern wir in der Perspektive der Subjektivierung von Arbeit die Folgen der hybriden betrieblichen Herrschaftsform subjektivierter Taylorisierung (2). Dic gesellschaftlichen Folgen diskutieren wir im abschließenden Abschnitt des Beitrags als forcierte Konkurrenz im Modus entfremdeter Selbstoptimierung (3).

\section{Das Management von Call-Center-Arbeit}

Call Center sind als Grenzstelle (vgl. Holtgrewe 2001) zwischen Unternehmen und Kunden eine Form der Rationalisierung von Dienstleistungsarbeit auf der Basis integrierter Informations- und Kommunikationstechnologien. Ihre Aufgabe ist die Bearbeitung von Kundenanliegen, gewöhnlich im Telefonat zwischen Agents $^{4}$ und Kunden, und die parallele systemkompatible Bearbeitung von Kundendaten im Informationssystem des Unternehmens. Die Arbeitsleistung wird durch eine Vielzahl von quantitativen und qualitativen Parametern, die z.B. die Anzahl und die Dauer der Telefonate vorgeben, definiert und im Zuge einer informatisierten Kontrolle vermessen.

Über die so vorstrukturierte technisch-dialogische Beziehung hinaus unterliegt der Dienstleistungsprozess weiteren, allgemein gültigen kommunikativen Regeln und sozial geteilten Erwartungen, die häufig zu Abweichungen von den idealtypisch vormodellierten Kommunikationssituationen führen: Kunden beharren auf Beschwerden, haben Informationslücken oder wollen sich nicht so einfach mit schnellen Lösungen abspeisen lassen. Diese Situation erzeugt betrieblicherseits Probleme hinsichtlich Effizienz und Effektivität der Dienstleistungsprozesse und des Personaleinsatzes. Hinreichende Kapazitäten (technischer wie personeller Art) sind zu garantieren; das Personal muss fachlich versiert, kommunikativ kompetent und emotional belastbar sein. Solche personalen Qualitäten sind zudem auftragsspezifisch stetig weiter zu entwickeln und müssen angesichts hoher Fluktuation auch Novizen angedient werden.

Zusammengenommen stehen Call-Center-Manager also vor dem doppelten Problem, sowohl Quantität wie Qualität der offerierten Dienstleistung durch arbeitsorganisatorische Maßnahmen zu sichern. Dazu stehen mehrere Instrumente zur Verfügung:

3 Der Beitrag fußt auf dem DFG-Projekt „Autonomie und Standardisierung in medienvermittelter Grenzstellenarbeit: Informatisierte Kommunikationsarbeit in Communication Cen" Genzstlenabeit. Infort tern an der TU Chemnicz. Ehoben wirden 117 nnterwews in sieben Call Centern unterschiedlicher Branchen an verschiedenen, städtisch geprägten Standorten. In Konzentration auf strukturelle Momente wird hier auf detaillierte Beschreibungen der Unternehmen verzichtet (vgl. dazu Matuschek u.a. 2007).

4 „Call Center Agent", kurz „Agent“, ist die übliche, ursprünglich englische, Bezeichnung für die unmittelbar am Telefon Arbeitenden in Call Centern. 
Erstens. Hinsichtlich der Arbeitszeiten dominieren zentralistische Modelle informatisierter Kapazitäts- und Schichtplanung. Dic Agents werden hier als prinzipiell während der gesamten Betriebszeit verfügbare Arbeitskraftäquivalente getaktet. Dieses Modell wird durch auf die einzelnen Arbeitssubjekte bezogene Nachsteuerung vor Ort optimiert: Dem operativ verantwortlichen Teamleiter obliegt die Administration individueller Arbeitszeiten, um aktuelles Anrufvolumen und Personaleinsatz zeitnah auszubalancieren. Telefonische Aufforderungen an die Mitarbeiter, außerplanmäßig zur Arbeit zu kommen bzw. gegebenenfalls früher zu gehen, zeugen von dieser mikropolitisch häufig mit Sanktionen (Verweigerung von Benefits, Zuweisung ungünstiger Arbeitszeiten etc.) besetzten Praxis. Es handelt sich um einen einseitigen Flexibilitätsgewinn der Unternehmen, während auf Seiten der Agents eher Belastungen im Verhältnis von Arbeit und Leben zu verzeichnen sind: arbeitszeitbezogene Zugeständnisse des Unternehmens (z.B. Teilzeit) werden mit dauerhafter Flexibilisierung der Arbeitssubjekte erkauft. ${ }^{5}$ Tendenziell wird die nach Dienstplan arbeitsfreic Zeit im eigenen Heim zur verlängerten Pause.

$Z$ weitens. Auch die Prozesse und Serviceangebote selbst werden zentral in damit befassten Fachabteilungen (Steuerungsgremien in Planung, Marketing und Technik) in Volumen und Inhalt vorbereitet und geformt. Die Call Center selbst sind ohne Mitspracherecht häufig bloße Empfänger von Anweisungen anderer Konzernteile. Mit sinkendem Grad der Komplexität der Dienstleistung steigt zudem generell die Regelungsdichte an. Zumeist ist in den Call Centern eine strikte Aufgabenspezialisierung der Arbeitstätigkeit vorherrschend, die nur gelegentlich durch Rotationsverfahren abgeschwächt wird. Formen des Job Enrichment (z.B. Delegation in Projekte) dienen intern als Motivationsinstrument und - angesichts einer strikt vertikalen Hierarchie und kaum vorhandener Aufstiegsmöglichkeiten - zugleich als Disziplinierungsinstrument: Das Management knüpft den Einsatz in solchen Projekten an die (jenseits des als selbstverständlich vorausgesetzten und informationstechnisch wie sozial kontrollierten Abarbeitens der vorgegebenen Anrufvolumina vorgenommene) Bewertung des Arbeitserfolgs, also z. B. anhand der mittels Vertragsabschlüssen belegbaren Rate der Transformation von Kundenkontakten in Dienstleistungskontrakte. Den unmittelbar vorgesetzten jeweiligen Teamleitern kommt in diesem Zusammenhang eine starke Stellung zu, womit sich nicht selten ein Verhältnis persönlicher Willfährigkeit der Agents zu ihnen einstellt.

Drittens. Die Qualität des sozialen Zusammenhalts unter der üblicherweise in Teams organisierten Belegschaft variiert zwischen rein formalen Zusammenschlüssen auf der einen und gemeinschaftlichen Gruppenstrukturen auf der anderen Seite. In kleineren Call Centern und in räumlich eng verbundenen

5 Diese Praxis fußt ganz wesentlich auf der Existenz von Kern- und teilzeitarbeitenden Randbelegschaften und verstärkt die Tendenz zur weitergehenden Spaltung der Belegschaften.
Teams dominiert ein enger Zusammenhalt zwischen den Agents; der Teamleiter in diesen Teams ist ein primus inter pares. Bei rein formaler Teambildung herrschen stärker hierarchieorientierte Steuerungsmodelle vor. Die innerbetriebliche Kommunikation passt sich diesen Strukturen an. Sie ist formal generell top-down organisiert und fungiert als reines Informationsmedium, lässt aber in unterschiedlichem Maß informelle Strukturen zu, die einer interaktiven Kommunikation förderlich und einem verbesserten Arbeitsklima dienlich sind. Zugleich erleichtert letzteres Vorgehen dem Management den Zugriff auf die Kommunikationserfahrung der Arbeitenden.

Viertens. Im Hinblick auf die Zielvorgaben und die Kontrolle der Arbeitsleistung agiert das Management überwiegend vertriebsorientiert. Auch wenn die formale Arbeitsleistung der Einzelnen permanent anhand unterschiedlicher $\mathrm{Pa}$ rameter überprüft wird, steht der Vertriebserfolg, also in der Regel Kauf von bzw. Vertragsabschlüsse über Dienstleistungen, letztlich im Zentrum der Bewertung der Arbeitskraft. Die nicht unerheblichen variablen Lohnbestandteile sollen demgemäß eine Orientierung an hohen Abschlussraten befördern. Die Bemessungsgrundlagen dafür werden mit dem Verweis auf den Markt extern legitimiert und zugleich als Instrument des Konkurrenzkampfes betont. Leistungsanreize verwandeln sich so in permanenten Leistungsdruck (vgl. Pohlmann/Grewer 2003). Dabei reduzieren sich die Vorgaben auf ein unbestimmtes, gerade dadurch aber die Selbstkontrolle der Arbeitenden stimulierendes ,immer mehr".

Nicht nur die reine Anzahl der Gespräche (bei der Telefonauskunft über 50 Gespräche pro Stunde) legt nahe, im Zusammenhang mit Call Centern von einer ,assembly line in the head" (Taylor/Bain, 1999: 101) zu sprechen. Auch die arbeitsorganisatorischen Rahmenbedingungen zeigen Parallelen zu tayloristisch-fordistischen Prinzipien betrieblicher Herrschaft. Die Zerstückelung von qualitativ singulären Kundendialogen in durchschnittlich quantitativ bemessene Kommunikationseinheiten erlaubt es, die systemgerechte Standardprozedur als Kern des Kundenservices zu etablieren und die Variabilität der Dienstleistung deutlich zu reduzieren. ${ }^{6}$ Die bis auf den einzelnen Kundenkontakt rückführbare Durchdringung mit quantitativen Messpunkten zeugt von einer hohen Kontrolldichte, die der oftmals beschworenen Autonomie in der Dienstleistungsarbeit entgegensteht. Häufig ausschließliche Single-ItemInterpretation der Kennziffern, die zudem selten über einen längeren zeitlichen Horizont verfügen, begründen dann als notwendig erachtete arbeitsorganisatorische Veränderungen durch das Management, oftmals ohne die Erfahrung der front-line-worker zu berücksichtigen.

6 Diese Logik befördert Tendenzen, Kunden in Konsumarbeiter zu verwandeln, die Teile der Dienstleistung selbst erbringen (vgl. Voß/ Rieder 2005). 
Personenbezogene Dienstleistung gerinnt, bei aller Variabilität der Einzelgespräche, zu einem in den Planungsabteilungen weitgehend vorstrukturierten Produkt - auch dies eine Parallele zur konventionellen Fließbandproduktion. Standardisierung greift zunächst auf Seiten des Dienstleistungsproduktes, in der Folge aber auch in der Arbeitsausführung um sich. Arbeitende können lediglich auf Standardlösungen zurückgreifen, die kaum noch individuelle Bearbeitung erlauben. Allenfalls verbale Kreativität wird zugelassen, soweit die Agents damit Standardlösungen rhetorisch in ein gutes Licht setzen können. Insoweit damit Authentizität, Hinwendung, Betreuung und ähnlich positiv konnotierte Gefühle erzeugt werden, handelt es sich offensichtlich um ein (lebensweltlich fundiertes) Korrektiv zur standardisierten Produktpalette. Den Kunden wird damit die Realität der prozessoptimierten Interaktion geschönt und die von ihnen erwartete Individualität des Services vorgegaukelt. Unter dem generellen Ziel rationalisierter Bearbeitung kontraktorientierter Interaktionen besteht damit ein funktional notwendiger betrieblicher Bedarf an subjektivierter Arbeit. Mit allgemeinen Leitlinien der Gesprächsführung in Verbindung mit Kennziffernsystemen wird die Dienstleistung managementseitig gerahmt, in Bezug auf die konkrete Ausgestaltung des einzelnen Gesprächs aber im Unbestimmten gehalten. Das Zugeständnis definierter Freiräume geschieht in der Erwartung eines flexiblen und der jeweiligen Situation angepassten Engagements der Arbeitenden. Ihr faktisches Handeln ist, vor allem bei ausbleibendem Erfolg, allerdings immer ex post sanktionierbar.

Neben den sprachlichen Freiheiten im direkten Dialog ist dies insbesondere im Hinblick auf das betriebliche Wissensmanagement bedeutsam: Tendenziell ziehen sich Betriebe aus Kostengründen auf die Bereitstellung der unbedingt erforderlichen Kontextinformationen zurück. Die Beschaffung von peripherem Kontextwissen obliegt damit allein den einzelnen Mitarbeitern. Die Sicherung beruflicher Expertise wie der Dienstleistungsqualität liegt so in weiten Teilen bei den Arbeitenden selbst. Technische wie soziale Kontrollmechanismen sichern den unmittelbar auftragsbezogenen Erfolg dieser Form der Wissensaneignung ab. Faktisch existiert damit ein reduziertes System betrieblicher Weiterbildung, das einseitig notwendige Produkt- und Prozessinhalte, nicht aber darüber hinaus gehende Qualifikationsmaßnahmen fokussiert.

Neben den bisher genannten Eigenheiten ist für viele Call Center insgesamt eine Führungskultur kennzeichnend, die weitgehende kennzifferndefinierte Globalziele voranstellt, ohne alle Einzelheiten des Arbeitsvollzugs zu berücksichtigen. Funktional wird der Arbeitsprozess daher erst durch eigenständige strukturierende Leistungen der arbeitenden Subjekte (vgl. Kleemann et al. 2002). Als Freisetzung der Subjekte aus fremdbestimmten Arbeitszusammenhängen gepriesen, zieht sich das Management letzten Endes aus der ureigenen Aufgabe der Arbeitsorganisation bei Konzentration auf Ergebnisvorgabe und -kontrolle zurück. Dass dabei konkrete Direktiven unterbleiben und auf eine (gerahmte) Selbststeuerung gesetzt wird, erhöht die Flexibilität, indem statische Befehlsketten reduziert werden. Insofern handelt es sich um eine Freigabe von nur solchen Steuerungsbereichen, die top-down zu organisieren als kontraproduktiv gilt.

Diese Managementpraktiken bedeuten eine Akzentverschiebung. Wurde schon vormals in funktionalistischer Manier mehr oder weniger am Regelwerk Taylors vorbei auf subjektive Fähigkeiten der Arbeitenden zurückgegriffen, so geschieht dies unter dem Zeichen subjektivierter Arbeit nun explizit und als ständige Anforderung: „die gezielte betriebliche Nutzung der Subjektivität der Arbeitenden wird offizialisiert" (Kocyba 2005, 80). Dazu greift das Management auf unterschiedlichste motivationale Techniken zurück: von der kennziffernbasierten Kontrolle über variable Entgeltsysteme bis hin zu vergemeinschaftenden Techniken (vgl. Kleemann/Matuschek 2003). Neben Belobigungen wird auch auf Effekte wie Skandalisierungen (vermeintlich unzureichender Leistungen) und öffentliche Verpflichtungen der Mitarbeiter gesetzt, die repressiven Charakter haben. Angesichts volatiler Märkte versprechen Flexibilitätsgewinne durch Subjektivierung einen Wettbewerbsvorteil. Den eindeutig erscheinenden Kennziffern steht die (kontrollierte) Unbestimmtheit des Arbeitshandelns zur Seite - in der Tendenz interessiert nur, was als Arbeitserfolg abzurechnen ist; die Arbeitsleistung verschwindet dahinter.

Im Mittelpunkt all dieser Bemühungen des Managementhandelns stehen avancierte Accounting-Konzepte (vgl. dazu Schmidt 2005; Vormbusch 2005). Dem liegt eine Rationalisierungsperspektive zu Grunde, mit der die marktzentrierte Ausrichtung einzelner Arbeitsplätze befördert wird. Partizipative Elemente, wie sie in Selbstverwirklichungs- bzw. Humanisierungs-Debatten noch zentral waren, sind dieser Form der Vernutzung subjektiver Kompetenzen fremd. Indem das Management auf positiv besetzte Leitbilder (wie Eigenständigkeit, Authentizität, Kreativität) rekurriert, die aber im betrieblichen Interesse kanalisiert und instrumentalisiert werden, erfolgt eine ideologisch überhöhte selektive Aktivierung von Fähigkeiten der Arbeitenden (vgl. Kleemann et al. 2002). Subjektpotenziale sollen also zur Entfaltung gebracht und gleichzeitig zugerichtet werden. Letztlich vollzieht sich eine „entsubjektivierende Subjektivierung": Mehr als bislang üblich sollen ausgewählte und eingrenzbare personale Qualitäten der Arbeitenden in die Arbeit eingebunden werden, die (ganze) Person des Arbeitenden mit ihren lebensweltlichen Bedürfnissen aber nach wie vor außerhalb der Erwerbssphäre verbleiben.

Gleichwohl könnte diese modernisierte Form des Taylorismus, die eine vermehrte Eigenständigkeit der Subjekte induziert, auch emanzipatorisches Potenzial besitzen, und das Management könnte die Geister, die es rief, nicht wieder los werden: Möglich ist, dass die Arbeitenden die Leitlinien des Managements wider Erwarten ernst nehmen, die Einhaltung der (rhetorischen) Zielsetzungen hinsichtlich der Kundenzufriedenheit einfordern und auf einer ent- 
sprechenden Arbeitsweise beharren, die dann auch dem eigenen emotionalen Haushalt zu Gute kommt. Allerdings sind solche Formen des impliziten Widerstands anders als offen ausgetragene Interessenkonflikte zwischen Kapital und Arbeit ihrerseits prinzipiell ausbeutbar - was wiederum auf die Widersprüchlichkeit des Prozesses der Subjektivierung von Arbeit verweist. Dem soll im Folgenden ausführlicher nachgegangen werden.

\section{Selbstorganisation mit Folgen: Forderungen der Subjekte}

Dass die skizzierten Prozesse von den arbeitenden Subjekten internalisiert und in ihr alltägliches Handeln eingebettet werden, ist viel beschworene Erkenntnis in der arbeitssoziologischen Diskussion. Sie werden als eine in ihren Ergebnissen ambivalent einzuschätzende Entwicklung (vgl. Kocyba 2000) angesehen, die „riskante Chancen“ (Lohr/Nickel 2005) eröffnet. Die Prozesse der Internalisierung des Marktes (Moldaschl/Sauer 2000) und der Subjektivierung von Arbeit verlaufen jedenfalls nicht bruchlos und frei von Widersprüchen:

Erstens. Call Center sind häufig Ausgründungen, in die zuvor im Mutterunternehmen beschäftigte Fachkräfte bei reduzierter Entlohnung verschoben werden. Angesichts der damit verbundenen Degradierung halten Betroffene strikt an ihrem erworbenen beruflichen Status und ihrer Berufsidentität fest. Zugleich legen sie in der Erwerbsdimension einen realitätsbezogenen Pragmatismus an den Tag: Sie „wählen" zwischen den Übeln der Erwerbslosigkeit und einer unterqualifizierten Tätigkeit Letzteres. Dabei werden innerbetriebliche und gesellschaftliche Wahrnehmungen von Call-Center-Arbeit reflektiert und die eigene Tätigkeit als Call-Center-Agent als Sackgasse, Sprungbrett oder Zwischenlösung bewertet. Der Rückgriff auf fachliche Expertise der grundständigen Ausbildung bezieht auch andere als marktförmige Kriterien (z.B. moralisch vertretbares Handeln als Techniker) ein. Dies kann (insbesondere in berufsbiographischer Langfristperspektive) zu einer eigenständigen Bewertung betrieblicher Ziele führen, wenn die Arbeitenden ihre berufsfachlichen Standards in die Arbeit einbringen und mit dem langfristigen Bedarf der Kunden entsprechende Dienstleistungen als Qualitätsmerkmal der eigenen Arbeit reklamieren, die in the long run dem Unternehmen zu Gute kommen. Auch wenn ihre Durchsetzungsmacht beschränkt ist, erzeugen die gegensätzlichen Orientierungen (z. B. Kosteneffizienz vs. Nachhaltigkeit) latente Konflikte und setzen das Management in ungewohnten Rechtfertigungszwang (zur Legitimation des Marktregimes vgl. Menz 2005).

Zweitens. Für die Arbeitsorientierung der Agents spielt angesichts kommunikativer Zumutungen von Kundenseite die nachhaltige Sicherung der eigenen Würde und persönlichen Integrität eine große Rolle. Daran entzünden sich Auseinandersetzungen mit dem Management um die Qualitätskriterien der Dienstleistungserbringung, aber auch um die Belastungen der auf Dauer mo- notonen Kommunikationsarbeit. Die von den Agents eingebrachten Qualitätsstandards beruhen häufig auf informellen Situationsdefinitionen gemeinsam mit den Kollegen des eigenen Teams und stellen kollektive Ansichten dar. Aufforderungen des Managements zur erhöhten Leistungsbereitschaft beziehen sich dagegen zumeist in vergleichsweise abstrakter und ideologisierender $\mathrm{Ma}$ nier auf das Unternehmen als ganzes in seiner Marktposition. Damit existieren in gewisser Weise betriebliche Parallelwelten, die erst zu einer produktiven Koexistenz finden müssen. Eine auf erhöhte Motivation zielende, aber bloß formal bleibende Vergemeinschaftung per Teambildung ist jedenfalls zum Scheitern verurteilt (vgl. Kleemann/Matuschek 2003).

Drittens. Die Arbeitspraktiken changieren zwischen dem Befolgen der arbeitsorganisatorisch gesetzten Vorgaben und kreativen Abwandlungen (v.a. im Kundenkontakt), mit denen betriebliche, aber auch individuelle Ziele durch die Subjekte verfolgt werden. Insoweit informelle Übereinkünfte eigensinnige und zum Teil deviante Praktiken tolerieren, zieht sich „Herrschaft durch $\mathrm{Au}$ tonomie" (Moldaschl/Sauer 2000, vgl. auch Wolf 1999) auf eine beobachtende, gegebenenfalls ex post sanktionierende Ebene zurück. Steht der betriebliche Rahmen dermaßen als individuelles Handlungsfeld offen, weiten die Subjekte diese Autonomie über den vom Unternehmen intendierten Bereich aus. Indem sie auf diesem Wege ihren eigenen Arbeitsstil (vgl. Kleemann/Matuschek 2001; Matuschek u.a. 2002) und eigene Werte und Vorstellungen einbringen, positionieren sich die Einzelnen gegenüber allen anderen Personen als eigenwillige Akteure im Handlungssystem betriebsförmiger Arbeit. Auch wenn sie dies nicht auf gleicher Augenhöhe mit Vorgesetzten vermögen (vgl. dazu Crozier/Friedberg 1993), zielt diese Positionierung darauf, unter den gegebenen Bedingungen ein auf sich selbst bezogenes stimmiges Verhältnis von betrieblichen Anforderungen und individueller Entäußerung zu finden.

Viertens. Im Hinblick auf Dienstleistungsorientierung und Kommunikationsarbeit nutzen die Subjekte ihre exklusive Position an der Grenzstelle und setzen in doppelter Hinsicht ihren Expertenstatus ein: Intern kommunizieren sie Ansprüche der Kunden in den Betrieb und nutzen diese zugleich als Argument für eine veränderte (i.e.S. weniger vertriebsorientierte) Kommunikationsarbeit. Extern konstituieren sie die Kunden als Laien und etablieren so ein hierarchisches Gefälle zu den Kunden, um vor eventuellen Zumutungen geschützt zu sein. Gegenüber dem Management gelingt es ihnen, die eigene Akteursmacht auszudehnen und eigene Vorstellungen über gelungene Gespräche einzubringen. Zugleich erzielen sie so einen Schutz vor psychosozialen Belastungen. Die Kommunikation gewinnt dadurch für die Arbeitenden einen zu beiden Seiten wichtigen Wert, indem sie von einer Aufgabe zu einem strategischen Faktor aufgewertet wird. „Reden kann jeder - kommunizieren nicht ${ }^{68}$ könnte das Credo dieser elitären Auffassung von der eigenen Arbeit als Kommunikationsexperten lauten. Damit definieren sich die Arbeitenden weniger 
als Ausführende betrieblicher Vorgaben, sondern als flexible Interpreten des unternehmerischen Willens in wechselnden Situationen.

Auf diese Situation hat das Management mit der Aussicht, Commitment zu produzieren, zu reagieren und entsprechende, die individuelle Befindlichkeit berücksichtigende Führungsstile zu etablieren. In der Perspektive der Subjektivierung von Arbeit (vgl. dazu Kleemann et al. 2002, Lohr/Nickel 2005; AG SubArO 2005) gewinnt damit im Hinblick auf die handelnden Personen insbesondere die reklamierende Subjektivität (vgl. Kleemann et al. 2002) einen zentralen Stellenwert: die Arbeitenden erheben eigene Qualitätsmaßstäbe, übertragen Standards der Kundenkommunikation auf die eigene Situation und leiten daraus Ansprüche ab, pochen auf ihre authentisch erprobte Expertise in der Kritik managementseitiger Entscheidungen u.a.m. Insgesamt zielen die Ansprüche darauf, als Person ernst genommen zu werden und die eigenen Erfahrungen in den Prozess des Arbeitens einbringen zu können, ohne als bloß Ausführende bewertet zu sein.

\section{Subjektivierte Taylorisierung}

Schlussfolgerungen zum Wandel der Arbeit

Derartige Ansprüche sind von den Betrieben instrumentalisierbar. Es kann daher nicht verwundern, dass der Terminus Subjektivierung, von einer analytischen Kategorie zum positiven Leitbild umdefiniert, Eingang in die Managementliteratur und den betrieblichen Alltag gefunden hat (vgl. Kocyba 2005). In der Praxis verschmelzen solche auf die Subjektivität der Arbeitenden ziclenden Führungsstrategien des Managements mit 'klassischen' Prinzipien des Scientific Management: Die tayloristische Trennung von Planung und Ausführung und die rigide Überwachung der Arbeitsausführung durch das Management bleiben unverändert bestehen. Dagegen wird der klassische tayloristische Modus der Kontrolle von Arbeit an einer zentralen Stelle geöffnet - und gewissermaßen auf höherer Ebene wieder geschlossen: Die Öffnung bezieht sich auf das tayloristische Prinzip der präzisen Vorgabe genauer Schritte des Arbeitsvollzugs, das im Kontext der Kommunikationsarbeit nur begrenzt praktikabel erscheint und durch Rahmenvorgaben ersetzt wird. Diese sollen die Arbeitenden in begrenzter Weise selbstorganisiert und individuell unter Einbringung ihrer Subjektivität ausfüllen. Die Schließung auf höherer Ebene vollzieht sich über eine Kontrolle sowohl der Performanz der Call-Center-Agents im Kundenkontakt (entlang vorgegebener Leitlinien der Kommunikation) als auch der objektivierbaren Arbeitsergebnisse (entlang von Kennziffern), die gewährleistet, dass die Agents ihre Subjektivität im Sinne der Unternehmensziele einsetzen. Arbeitsprozesse in dieser Form zu organisieren ist mit dem Terminus subjektivierte Taylorisierung treffend charakterisierbar.

Subjektivierte Taylorisierung beschränkt sich bezüglich der konkreten Ar- beitsausführung also auf die Formulierung von Globalzielen, die allerdings im Ergebnis noch individuell kontrollierbar und sanktionierbar sind. Den Arbeitenden wird zwar das Handwerkszeug zur Verfügung gestellt'; wie sie damit umgehen, ist ihnen in einem begrenzten Rahmen selbst überlassen. Die Befähigung, die aufgetragenen Aufgaben auch zufrieden stellend zu bewältigen, ist insofern ebenfalls von den Arbeitenden selbst aufrecht zu halten, als sie sich peripheres Produkt- wie Prozesswissen aus bereitgestellten Informationspools oder externen Quellen selbst beschaffen müssen. Steuerbar ist dies alles durch kennzifferngestützte Kontrolle, die um soziale Kontrollmechanismen innerhalb der teamförmigen Struktur noch erweitert wird. Zusammen erzeugen sie ein ständiges Interventionsrecht des Managements.

Dem Zwang, die zugemuteten Freiräume auszufüllen, steht bei Mitarbeitern der Wunsch danach zur Seite, 'interessante $e^{c}$ d.h. weniger repetitive Tätigkeiten auszufuhren, an arbeitsorganisatorischen Entscheidungen beteiligt zu werden und sich mit der Arbeit identifizieren zu können. Vor dem Hintergrund der eigenen beruflichen Erfahrungen werden entsprechende Forderungen, zum Teil aufgeladen mit moralischen Maßstäben, an die Arbeit an den Betrieb gestellt. Soweit diesen Anliegen aus subjektiver Sicht entsprochen wird, ist die Bereitschaft groß, auch inhaltlich über den eigentlichen Aufgabenbereich hinaus gehende, qualifizierte Mehrarbeit zu leisten. Offensichtlich kompensiert die in den Augen der Belegschaften erhöhte Qualität die Motivation zur Arbeit, auch über vertraglich fixierte Volumina hinaus. Insgesamt ist dies als ein Prozess des Ausbalancierens eigener Ansprüche an die Arbeit zu verstehen, der ein hohes Maß an Sinnstiftung transportiert.

Hier liegt nun auch eine Krux subjektivierter Taylorisierung: Indem subjektive Leistungen in der Arbeitspraxis gefordert und gefördert werden, erscheint diese Entwicklung als tendenziell unwiderrufbar. Insoweit dies als Abbau reglementierender Beschränkungen begrüßt und eine Bereitschaft entwickelt wird, immer weitere dieser Qualitäten dem Unternehmen zur Verfügung zu stellen, hat dies zwei Konsequenzen: Einerseits werden die Unschärfe der Arbeitsorganisation ebenso wie die mannigfaltigen Kontrollen zunehmen; andererseits können die Arbeitenden positive Erfahrungen mit selbstorganisierter Arbeit nicht gänzlich negieren. Erzeugt wird eine Dynamik in Richtung subjektivierter Arbeit, mit der zugleich zumindest implizit auf eine Entsolidarisierung und Vereinzelung der Arbeitenden gesetzt wird. Im Zuge der Vermarktlichung des einzelnen Arbeitsplatzes kommt der individuellen Selbstoptimierung bei einem nicht geringen Teil der Belegschaften eine höhere Wertigkeit zu als kollektiven

7 Integrierte Telefon- und Computertechnits strukturiert und kontrolliert die Arbeit dabei gleichermaßen: Anliegen der Kunden werden generell in standardisierte Datenbanken übertrachermaßen: Anliegen der Kunden werden generell in standardisierte Datenbanken übertra-
gen, womit Kommunikationsinhalte, Merkmalsausprägungen und oft auch der Kommunikationsverlauf vorgeprägt ist. Zugleich erlauben Datenprotokolle eine minutiöse Kontrolle der Arbeitenden. 
Interessen und werden letztere als der eigenen Produktivität hinderliche Begrenzungen aufgefasst.

Im soziologischen Diskurs ist umstritten, welche Arbeitenden und Branchen wie von den Prozessen der Subjektivierung von Arbeit betroffen sind (vgl. zusammenfassend: Dörre/Röttger 2003) und wem sich dies eher als Verlust von Sicherheit oder als Zugewinn an Autonomie darstellt. Als relativ weit fortgeschritten werden Prozesse der Subjektivierung und Entgrenzung in höher qualifizierten bzw. akademischen und künstlerischen Berufen eingeschätzt. Hier gehören Selbstorganisation und Eigenkontrolle seit langem zum Berufsbild, wenn auch in spezifischen Berufen unterschiedlich stark ausgeprägt und zum Teil mit Prekarisierungsfolgen behaftet. Andererseits werden Tendenzen der Re-Taylorisierung ausgemacht und insbesondere ein Abbau partizipativer Elemente beobachtet (vgl. Bosch 2000). Mithin ist davon auszugehen, dass es verfehlt wäre, eindeutige und unumkehrbare Trends anzunehmen. Dies heißt auch, überzogene Dramatisierungen zu vermeiden und die zum Teil widersprüchliche Pluralität von Entwicklungen zum Ausgangspunkt zu nehmen.

Die Diagnose einer subjektivierten Taylorisierung fokussiert solche ambivalent anmutenden Prozesse, in denen vom Management probate Mittel der fremdbestimmt-industriellen Arbeitsorganisation mit für die Betriebe positiven Effekten einer verstärkt selbstorganisierten Arbeit vereint werden. Es handelt sich hierbei um eine kontrollierte Öffnung, die gleichwohl wesentliche Leitlinien des Taylorismus beibehält. Kleinteilige Kontrollmechanismen wirken ebenso fort wie hoch arbeitsteilige Prozesse, die eingeforderten personalen Kompetenzen der Person sind nur in ihrer für den Dienstleistungsprozess funktionalen Qualität relevant. Die damit einher gehende Flexibilisierung der Arbeitszusammenhänge stellt gleichsam eine modernisierte Betriebsführung dar. Flecker (2005) verweist darauf, dass dies nicht nur als eine Reaktion auf veränderte Bedingungen in Käufer- und Absatzmärkten, sondern auch als Auswirkung gestiegener Renditeerwartungen von Aktionären und letztlich auch als Machtfrage in den Arbeitsbezichungen zu verstehen ist.

Sauers (2003) Perspektive entsprechend, dass die Entwicklungen im Bereich der Dienstleistungsarbeit nicht nur für diese selbst, sondern für einen Formwandel der gesellschaftlichen Verfasstheit von Arbeit insgesamt stehen, zeigt das Phänomen subjektivierter Taylorisierung in Call Centern, dass sich auch in mittelqualifizierten Tätigkeiten zunehmend eine marktbezogene Öffnung für arbeitsorganisatorische Vorgaben vollzieht. Entsprechende Anforderungen erfassen immer mehr Beschäftigtengruppen. Die Qualität subjektivierter Taylorisierung besteht nun nicht nur darin, den Subjekten mehr Freiräume (insbesondere kommunikativer Art) zuzugestehen oder teamförmige Rituale in die Führungskultur aufzunehmen. Die transformatorische Kraft, die dem subjektivierten Taylorismus innewohnt, ist eher auf der ideellen Ebene zu erkennen: Ganz offensichtlich können Manager mit dem Hinweis auf erforderliche per- sonale Kompetenzen erfolgreich an emanzipatorische Diskurse anschließen, faktisch damit aber erhöhten Anforderungen und einem erweiterten Zugriff auf das Privatleben der Beschäftigten den Weg bereiten. Zugleich findet in der Konzentration auf den Erfolg eine Entkopplung von Leistung und Entlohnung statt, die den marktzentrierten Wettbewerbsgedanken bis auf den einzelnen Arbeitsplatz herunter bricht.

Dergestalt flexibilisierte, d.h. in der Regel ausgeweitete Verfügungsgewalt über den Faktor Arbeit manifestiert ein tiefer gehendes Herrschaftsverhältnis, das eine spezifische Form der Entfremdung hervor bringt: Indem personale Kompetenzen entäußert werden, entfremden sich die Arbeitenden nicht nur vom Produkt und von der Tätigkeit, sondern partiell auch von ihren ureigenen individuellen Qualitäten, die sie als Person ausmachen. Und paradoxer Weise gilt: je weiter sich die Arbeitenden einbringen, desto mehr laufen sie Gefahr, entfremdet zu werden. Die im Sinne von Einforderungen der Subjekte gegenüber der Arbeit gefasste "normative Subjektivierung der Arbeit" (vgl. Baethge 1991) wird zum expliziten normativen Anforderungsprofil: Der eigene Wunsch danach, bei der Arbeit Spaß zu haben, verkehrt sich nun in die Aufforderung von außen, mit Spaß bei der Arbeit zu sein.

Mit der vom Management geforderten (und kontrollierten) Selbstorganisation der Arbeitenden scheinen aber auch veränderte Erwartungen der Beschäftigten an Partizipation einher zu gehen. Die Beschäftigten beanspruchen etwa Mitsprache bei Planung und Kontrolle oder fordern vom Unternehmen bei gleichzeitiger Akzeptanz seiner Flexibilitätsvorstellungen eine entsprechend flexible Berücksichtigung eigener individueller Wünsche. Hier deutet sich eine unintendierte Folge der unternehmensseitig forcierten partiellen Subjektivierung an: Freigesetzt wird tendenziell die ganze Persönlichkeit - zumindest aber mehr Anteile, als vom Management wirklich gewünscht. Der Prozess der Subjektivierung von Arbeit entwickelt also eine gewisse Eigendynamik, die sich nicht immer mit der manageriellen Zielsetzung, Subjektivität ausschließlich im betrieblichen Verwertungsinteresse zu nutzen, decken. Vielmehr eröffnet sich ein neuartiges "contested terrain", über dessen konkrete Ausgestaltung implizite oder explizite Aushandlungen zwischen Management und Arbeitenden entscheiden.

Die Beibehaltung des tayloristischen Grundgerüstes aus Kontrolle und Entlohnung ist in diesem Zusammenhang nicht als bloßes Überbleibsel überkommener Managementpraktiken anzusehen. Vielmehr dient dieses als eine Art vorgezogener Schutzwall gegenüber den von Baethge (1991) vermuteten emanzipatorischen Potenzialen einer Subjektivierung von Arbeit: Qualitativ wie quantitativ belastende Arbeit unter den Bedingungen eines personalisierten Wettbewerbs dürfte es zumindest erschweren, kollektive Forderungen nach wirklicher Autonomie zu stellen. Die rigiden Vorgaben dienen so in gewisser Weise der Domestizierung subjektiviert Arbeitender. 
Die Dominanz der Kennziffern als Kontrollinstanz in Verbindung mit direkter und bewusst als Sozialtechnologie eingesetzter Konkurrenz zwischen den Arbeitenden sowie der Anforderung an eine permanente Optimierung und Steigerung der eigenen Arbeitsleistungen befördert tendenziell eine Vereinzelung der Arbeitenden, die sich dem schwerlich mit dem Verweis auf erbrachte „hinreichende ${ }^{*}$ Arbeitsergebnisse entzichen können. Auch teamförmige Strukturen dienen als wechselseitige Kontrollinstanz der Arbeitenden. Etabliert hat sich eine vom realen Arbeitsprozess entkoppelte Ideologie der ständigen Optimierung (vgl. Bröckling 2007), die das Halten eines Niveaus als Stillstand abwertet. In dieser Kultur sind die Einzelnen aufgefordert, sich permanent selbst zu optimieren und an der Verbesserung ihrer Leistung zu arbeiten. Teile der Arbeitenden haben sich diese Ideologie zu eigen gemacht und profitieren von Ausschüttungen, Sonderprämien etc., an denen der eigene Erfolg materiell spürbar wird. Andere bringen sich durch eigene Leistungsvorstellungen ein (vgl. dazu auch Menz 2005). Gelegentlich sind daher Entsolidarisierungstendenzen zu verzeichnen, die von Konflikten zwischen Kollegen bis hin zur Infragestellung begrenzender betrieblicher Vereinbarungen reichen. Instanzen der Interessenvertretung sehen sich hier vor die Herausforderung gestellt, divergierenden Interessen der Beschäftigten entsprechen zu müssen. Gelingt dieses nicht, ist ein Machtverlust kollektiver Gremien zu Gunsten individualisierter Aushandlungen zu erwarten. Auf lange Sicht könnten damit Pfade hin zu einem atomisierenden Kapitalismus geebnet werden, in dem isolierte Einzelkämpfer um Arbeitsaufträge ihres Arbeitgebers konkurrieren müssen und so zum „Spielball der Gelegenheitsstrukturen“ (Wagner 2005: 177) werden. Prekarität dürfte dann zur Normalität subjektiviert Arbeitender gehören (vgl. Dörre 2005). Dass damit Herrschaft stabilisierende Folgen einhergehen, dürfte evident sein und von Managern als natürliche Begrenzung allzu subjektivierter (und mithin emanzipatorischer) Ansprüche durchaus begrüßt werden. Immer hin könnte mittels solcher Konkurrenzen auch zukünftigen Entspannungen auf dem Arbeitsmarkt begegnet werden, dessen gegenwärtige Konstitution Gelegenheit bietet, Deregulierungen und Flexibilisierungen durchzusetzen.

\section{Literatur}

AG SubArO (2005): Ökonomie der Subjektivität - Subjektivität der Ökonomie. Berlin: edition

sigma.
Baethge, Martin (1991): Arbeit, Vergesellschaftung, Identität - Zur zunehmenden normativen Subjektivierung von Arbeit. In: Soziale Weit 42 (1), 6-19

Baukrowitz, Andrea; Boes, Andreas (1996): Arbeit in der "Informationsgesellschaft". Einige Überlegungen aus einer (fast schon) ungewohnten Perspeltive. In R Schmiede (Hg.) 129 - 157.

leg -252 .

Bosch, Gerhard (2000): Arbeitszeit und Arbeitsorganisation. Zur Rolle von Produkt- und Arbeitsmärkten im internationalen Vergleich. Arbeit, 3, 175 - 190.
Bröckling, Ulrich (2007): Das unternehmerische Selbst. Soziologie einer Subjektivierungsform Frankfurt a.M.: Suhrkamp

Crozier, Michel/ Friedberg Erhard (1993): Die Zwänge kollektiven Handelns. Über Macht und Organisation. Frankfurt a. M.: Hain

Dörre Klaus (2005): Prekäre Beschäftigung - ein unterschätztes Phänomen in der Debatte um die Marktsteuerung und Subjektivierung von Arbeit In: K. Lohr; H. M. Nickel (Hg.), $180-206$.

Dörre Klaus/ Röttger, Bernd (2003): Das neue Marktregime. Hamburg: VSA

Flecker, Jörg (2005): Interne Flexibilisierung - von der Humanisierungsvermutung zum Risikobefund. In: M. Kronauer; G. Linne (Hg.), Flexicurity. Die Suche nach Sicherheit in der Flexibilität, 73 - 93. Berlin: edition sigma

Gottschall, Karin; Voß G. Günter (Hg.) (2003): Entgrenzung von Axbeit und Leben. Zum Wandel der Beziehung von Enwerbstätigkeit und Privatsphäre im Alltag. München/Mering: Hampp.

Holtgrewe, Ursula (2001): Organisationsdilemmata und Kommunikationsarbeit. Call Center als informatisierte Grenzstellen. In: I. Matuschek et al., 55 - 70.

Joas, Hans (1992): Die Kreativität des Handelns. Frankfurt a.M.: Suhrkamp

Kleemann, Frank; Matuschek, Ingo (2001): Zur Erfassung subjektiver Leistungen in informatisierter Arbeit. In: Matuschek et al., 257 - 279

Kleemann, Frank; Matuschek, Ingo (2003): Subjektivierung in Informatisierter Kommunikationsarbeit: Manufacturing Consent in High Quality Call Centern. In: K. Schönberger; S. Springer (Hg.), Subjektivierte Arbeit. Mensch, Organisation und Technik in der entgrenzten Arbeitswelt, 117 - 142. Frankfurt a. M.: Campus.

Kleemann, Frank; Matuschek, Ingo (2008 [i.E.]): Informalisierung als Komplement der Informatisierung von Arbeit. In: Ch. Funken; I. Schulz-Schaeffer $(\mathrm{Hg}$.), Digitalisienung der Arbeitswelt Die Neuordnung formaler und informeller Prozesse in Unternehmen, Wiesbaden: VS Verlag.

Kleemann, Frank; Matuschek, Ingo; Voß, G. Günter (2002): Subjektivierung von Arbeit. Ein Ü berblick zum Stand der Diskussion. In: M. Moldaschl; G. G. Voß (Hg.), Subjektivienung von Arbeit, 53 - 100. München: Hampp

Kocyba, Hermann (2000): Der Preis der Anerkennung. Von der tayloristischen Missachtung zur strategischen Instrumentalisierung der Subjektivität der Arbeitenden. In: U. Holtgrewe; S. Strategischen Instrumentalisierung der Subjektivitat der Arbeitenden. In: U. H. Wagner (Hg.), Anerkennung und Arbeit, 127 - 140. Konstanz: UVK.

Kocyba, Hermann (2005): Selbstverwirklichungszwänge und neue Unterwerfungsformen. Paradoxien der Kapitalismuskritik. In: AG SubArO ( $H g$.), 79 - 93. Berlin: edition sigma.

Kratzer, Nick (2003): Arbeitskraft in Entgrenzung. Grenzenlose Anforderungen, enweiterte Spiel räume, begrenzte Ressourcen. Berlin: edition sigma.

Kropf, Julia (2005): Flexibilisierung - Subjektivierung - Anerkennung. Auswirkungen von Flexibilisierungsmaßnahmen auf die Anerkennungsbezichungen in Unternehmen. München: Biblion.

Lohr, Karin; Nickel, Hildegard Maria (2005): Subjektivierung von Arbeit - Riskante Chancen. In dies. $(\mathrm{Hg}$ ), Subjektivierung von Arbeit. Riskante Chancen, 207 - 239. Münster: Westf. Dampfboot.

Matuschek, Ingo; Henninger, Annette; Kleemann, Frank (Hg.) (2001): Neue Medien im Arbeitsalltag. Empirische Befunde - Gestaltungskonzepte - Theoretische Perspektiven. Wiesbaden: WV Matuschek, Ing; Kleemann, Frank; Voß, G. Günter (2002): Personaler Arbeitsstil. Ein Konzept zur Untersuchung 'subjektivierter' Arbeit. In: I. Moldaschl; G.Voß, 219 - 238

Matuschek, Ingo; Arnold, Katrin; Voß, G. Günter (2007): Subjektivierte Taylorisierung. Organisation und Praxis medienvermittelter Dienstleistungsarbeit. München: Hampp

Menz Wolfgang (2005): Das Subjekt der Leistung und die Legitimität des Marktregimes. In: $A G$ SubArO (Hg.), 95-116. Berlin: edition sigma

Minssen, Heiner (2000): Begrenzte Entgrenzungen - Wandlungen von Organisation und Arbeit Berlin: edition sigma.

Moldaschl, Manfred; Sauer, Dieter (2000): Internalisierung des Marktes - Zur neuen Dialektik von Kooperation und Hersschaft. In: H. Minssen (Hg.), $205-224$.

Moldaschl, Manfred; Voß, G. Günter (Hg.) (2002, [2005]): Subjektivierung von Arbeit. München/ Mering: Hampp

Pohlmann Markus; Grewer, Hans-Günter (2003): Dienstleistungsarbeit im Zeichen von Vermarktlichung und neuer Leistungsorientierung. In: Pohlmann et al., $295-305$. 
Pohlmann, Markus; Sauer, Dieter; Trautwein, Gudrun; Wagner, Angelika (Hg.) (2003): Dienstleistungsarbeit: Auf dem Boden der Tatsachen. Befunde aus Handel, Industrie, Medien und ITBranche. Berlin: edition sigma.

Pongratz, Hans J.; Voß, G. Günter (1997): Fremdorganisierte Selbstorganisation. In: Zeitschrift für Personalforschung, 11 (1), $30-53$.

Sauer, Dieter (2003): Einleitung: Arbeit, Leistung und Interessenhandeln in der "tertiären" Organisation. Dienstleistungsarbeit als Forschungsfeld. In: M. Pohlmann et al., $15-25$

Schumann, Michael (2000): Industriearbeit zwischen Entfremdung und Entfaltung. In: SOFI Mitteilungen 28, $103-112$

Schimank, Uwe (1986): Technik, Subjektivität und Kontrolle in formalen Organisationen - ein Theorieperspeltive. In: R. Seltz; U. Mill; E. Hildebrandt (Hg.), Organisation als soziales System. Kontrolle und Kommunikationstechnologie in Arbeitsorganisationen, 71 - 91. Berlin: dition sigma.

Schmidt, Angela (2005): Rentiere ich mich noch? Activity Based Costing und seine Wirksamkeit im Tun der Beschäftigten. In: H. Wagner (Hg.), 131 - 153. Hamburg: VSA

Schmiede, Rudi (1996) (Hrsg.): Virtuelle Arbeitswelten. Arbeit, Produktion und Subjekt in der „Informationsgesellschaft". Berlin: edition sigma.

Taylor, Frederic W. (1977 [1919]): Die Grundsätze wissenschaftlicher Betriebsfuihrung. Weinheim: Beltz.

Taylor, Phil/ Bain, Peter, (1999): „An assembly line in the head": work and employee relations in the call centre. In: Industrial Relations Journal, $30(2), 101-117$

Vormbusch, Uwe (2005): Das neue Alphabet des Kapitalismus: von A wie Audit bis $Z$ wie Zertifizierung. In: $H$. Wagner, $87-112$.

Voß, G. Günter (1998): Die Entgrenzung von Arbeit und Arbeitskraft. Eine subjektorientierte Interpretation des Wandels der Arbeit. In: Mitteilungen aus der Arbeitsmarkt- und Berufsforschung, 31 (3), 473 - 487.

Voß3. G. Günter; Pongratz, Hans, J. (1998): Der Arbeitskraftunternehmer. Eine neue Grundform der „Ware Arbeitskraft"? In: Kölner Zeitschrift für Soziologie und Sozialpsychologie, 50 (1), $131-158$.

Voß, G. Günter; Rieder, Kerstin (2005): Der arbeitende Kunde. Wenn Konsumenten zu unbezahlten Mitarbeitern werden. Frankfurt a.M.: Campus.

Wagner, Hilde (2005): „Die Macht der Zahlen“: Neue Steuerung im Betrieb. In: dies. (Hg.), 9 - 22. Wagner, Hilde (Hg.) 2005: „Rentier" ich mich noch?" Neue Steuerungskonzepte im Betrieb. Hamburg: VSA.

Wallraff, Günter (2007): Undercover im Call Center. In: ZEITmagazin Leben, 22, 24.05.2007, 16-25

Weltz, Friedrich (1991): Der Traum von der absoluten Ordnung und die doppelte Wirklichkeit der Unternehmen. In: E Hildebrandt ( $\mathrm{Hg}$ ), Betriebliche Sozialverfassung unter Veränderungsdruck, 85-97. Berlin: edition sigma.

Wolf, Harald (1999): Arbeit und Autonomie. Ein Versuch über Widersprüche und Metamorphosen kapitalistischer Produktion. Münster: Westfälisches Dampfboot.

\section{Mario Candeias}

\section{Die neuen Solo-Selbständigen zwischen Unternehmergeist und Prekarität}

Eine selbständige Tätigkeit aufzubauen wird als „Existenzgründung“ bezeichnet, als ob die Betreffenden zuvor keine gehabt hätten. Der Austritt aus der Lohnarbeit, aus einer vermeintlich unverantwortlichen, abhängigen Existenzweise, erscheint als Übernahme individueller Eigenverantwortung und Gewinnung von Unabhängigkeit. Als (Mikro)Unternehmer - und sei es nur meiner Selbst - gelte ich als gesellschaftlicher Leistungsträger und schaffe potenziell Arbeitsplätze (wenn auch nur meinen eigenen), statt als 'Arbeitnehmer' nur einen solchen zu besetzen. Selbst den abhängig Beschäftigten wird unternehmerisches Denken eingebläut. Das Unternehmen wird ideologisch zum Zentrum gesellschaftlicher Organisation, das als einziges produktive und zukunftsweisende Arbeitsplätze schaffe (tatsächlich werden nahezu 50 Prozent des Bruttoinlandsproduktes nicht im privat-kapitalistischen Sektor produziert und nach UN-Schätzungen 50 Prozent des globalen Reichtums als unbezahlte Reproduktionsarbeiten erbracht, zumeist von Frauen).

Nach Erhebungen des Sozialbeirats der Bundesregierung (Financial Times v. 28.11.07) gibt es in Deutschland 2,3 Mio. Solo-Selbständige (was 50 Prozent aller Unternehmen entspräche). Hinter der Betonung des Unternehmerischen in der Debatte verschwinden die Tätigkeit und die Verhältnisse, in denen diese Selbständigkeit sich bewegt. Diese Verschiebung der selbständig Arbeitenden in die ,symbolische und kulturelle Sphäre des kapitalistischen Unternehmens“ (Bologna 2006), statt in jene der gesellschaftlichen Arbeit und die damit verbundenen Widersprüche, fïgt sich in die große Erzählung vom Ende der Arbeit - gemeint ist: der Lohnarbeit. Durch die diskursive Eingemeindung breiterer gesellschaftlicher Gruppen erscheinen politische Forderungen der Unternehmer nicht als partikulare Klasseninteressen. ${ }^{1}$ Dagegen sollen hier Unterschiede und Gemeinsamkeiten der sog. Neuen bzw. Solo-Selbständigen im Verhältnis zur Lohnarbeit bestimmt werden. Trotz Differenzen bewirken die Veränderungen von Arbeits- und Lebensweisen dabei eine Konvergenz prekärer Lagen, sowohl bei den Solo-Selbständigen wie abhängig Beschäftigten. ${ }^{2}$

1 Zur neoliberalen Umdeutung und Erweiterung des Unternehmerbegriffs vgl. Plehwe (2007). 2 Spätestens seit der Studie aus dem Umkreis der Friedrich-Ebert-Stiftung (Müller-Hilmer 2006) zu Fragen der Ausbreitung von Prekarität, ist das Problem zu einem politischen ThePROKLA. Zeitschrift fur kritische Sozialwissenschaft, Heft 150, 38. Jg., 2008, Nr. 1, 65-81 